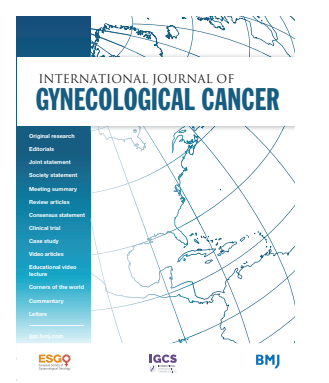

\title{
Randomized trial of primary debulking surgery versus neoadjuvant chemotherapy for advanced epithelial ovarian cancer (SCORPION-NCT01461850)
}

Anna Fagotti (D) , ${ }^{1,2}$ Maria Gabriella Ferrandina, ${ }^{1,2}$ Giuseppe Vizzielli, ${ }^{1}$ Tina Pasciuto, ${ }^{3}$ Francesco Fanfani, ${ }^{1,2}$ Valerio Gallotta, ${ }^{1}$ Pasquale Alessandro Margariti, ${ }^{1}$ Vito Chiantera, ${ }^{4,5}$ Barbara Costantini, ${ }^{1}$ S. Gueli Alletti, ${ }^{1}$ Francesco Cosentino, ${ }^{6}$ Giovanni Scambia ${ }^{1,2}$

- Additional material is published online only. To view please visit the journal online (http://dx.doi.org/10.1136/ijgc2020-001640).

For numbered affiliations see end of article.

Correspondence to Anna Fagotti, Woman, Child and Public Health Department, Fondazione Policlinico Universitario A. Gemelli, IRCCS, Roma 8 00168, Italy; annafagotti70@gmail.com

Received 20 May 2020 Revised 27 August 2020 Accepted 28 August 2020 Published Online First 7 0ctober 2020
Check for updates

(C) IGCS and ESGO 2020. № commercial re-use. See rights and permissions. Published by BMJ.

\footnotetext{
To cite: Fagotti A, Ferrandina MG, Vizzielli G, et al. Int J Gynecol Cancer 2020;30:1657-1664.
}

\section{HIGHLIGHTS}

- Primary debulking surgery and neoadjuvant chemotherapy have superimposable survival outcome in advanced epithelial ovarian, fallopian tube or primary peritoneal cancer patients with high tumor load.

- Primary debulking surgery and neoadjuvant chemotherapy have different toxicity outcomes.

- Residual tumor is the main prognostic factor at any surgery (either primary surgery or interval surgery).

\section{ABSTRACT}

Objective To investigate whether neoadjuvant chemotherapy followed by interval debulking surgery is superior to primary debulking surgery in terms of perioperative complications and progression-free survival, in advanced epithelial ovarian, fallopian tube or primary peritoneal cancer patients with high tumor load.

Methods Patients with advanced epithelial ovarian, fallopian tube or primary peritoneal cancer (stage IIIC-IV) underwent laparoscopy. Patients with high tumor load assessed by a standardized laparoscopic predictive index were randomly assigned (1:1 ratio) to undergo either primary debulking surgery followed by adjuvant chemotherapy (arm A), or neoadjuvant chemotherapy followed by interval debulking surgery and adjuvant chemotherapy (arm B). Co-primary outcome measures were progression-free survival and post-operative complications; secondary outcomes were overall survival, and quality of life. Survival analyses were performed on an intention-to-treat population.

Results 171 patients were randomly assigned to primary debulking surgery $(n=84)$ versus neoadjuvant chemotherapy $(n=87)$. Rates of complete resection (R0) were different between the arms $(47.6 \%$ in arm A vs $77.0 \%$ in arm $B ; p=0.001)$. 53 major postoperative complications were registered, mainly distributed in arm A compared with arm B (25.9\% vs $7.6 \% ; p=0.0001)$. All patients were included in the intent-to-treat analysis. With an overall median follow-up of 59 months $(95 \% \mathrm{Cl} 53$ to 64), $142(83.0 \%)$ disease progressions/recurrences and 103 deaths (60.2\%) occurred. Median progression-free and overall survival were 15 and 41 months for patients assigned to primary debulking surgery, compared with 14 and 43 months for patients assigned to neoadjuvant chemotherapy, respectively (HR $1.05,95 \% \mathrm{Cl} 0.77$ to 1.44 , $\mathrm{p}=0.73$; HR 1.12, $95 \% \mathrm{Cl} 0.76$ to $1.65, \mathrm{p}=0.56$ ).

Conclusions Neoadjuvant chemotherapy and primary debulking surgery have the same efficacy when used at their maximal possibilities, but the toxicity profile is different.

\section{INTRODUCTION}

The majority of patients with ovarian, fallopian, and primary peritoneal cancer are diagnosed with advanced disease. Two randomized multicenter trials have shown that neoadjuvant chemotherapy is non-inferior to primary debulking surgery for both progression-free and overall survival. ${ }^{12}$ Results from these studies are considered interesting but are limited, due to patient heterogeneity (disease stage and performance status), low cytoreduction rate in the primary debulking surgery arm, and low accrual per center.

Moreover, an individual patient meta-analysis of the European Organisation for Research and Treatment of Cancer (EORTC) 55971 and CHORUS trials has indicated that primary debulking surgery and neoadjuvant chemotherapy may have different efficacy in advanced epithelial ovarian cancer patients, according to disease extension. Indeed, neoadjuvant chemotherapy has been associated with better clinical outcome than primary debulking surgery in International Federation of Gynecology and Obstetrics (FIGO) stage IV patients, whereas patients with initial abdominal disease $<5 \mathrm{~cm}$ treated with primary debulking surgery had an increased survival rate when compared with those who underwent neoadjuvant chemotherapy. ${ }^{3}$

We designed the SCORPION trial (NCT01461850) to overcome limitations of the previous studies. ${ }^{4}$ Patients with epithelial ovarian cancer were eligible if diagnosed with advanced intra-abdominal disease assessed through a consolidated, reproducible, and 


\section{Original research}

reliable laparoscopic Predictive Index (PI) score. ${ }^{5}$ The trial was designed for a single institution (and its affiliates) with high accrual of patients per year and committed to maximal surgical effort. The study was designed to demonstrate that primary debulking surgery was associated with a higher peri-operative complication rate than interval debulking surgery after neoadjuvant chemotherapy. Subsequently, we aimed to determine whether there was significant improvement in progression-free survival in the neoadjuvant chemotherapy arm over the primary debulking surgery arm.

\section{METHODS}

The local ethical committee approved the trial. It was registered on https://clinicaltrials.gov (No. NCT01461850) and follows the ethical principles outlined by the Declaration of Helsinki. All patients gave written informed consent before starting the study. Trial management and progress were monitored by an independent data monitoring committee at the Catholic University of the Sacred Heart Clinical Trial Center.

\section{Patient Selection}

Patients aged 18 to 75 years with presumed FIGO stage IIIC or IV, Eastern Cooperative Oncology Group (ECOG) performance status $0-2$, and chemotherapy naïve were eligible. Other eligibility criteria included: estimated life expectancy of at least 4 weeks, adequate hematology, bone marrow, respiratory, hepatic, cardiologic, and renal function (creatinine clearance $>60 \mathrm{~mL} / \mathrm{min}$ according to Cockroft formula ${ }^{6}$ ). The life expectancy was clinically evaluated through the ECOG performance status, history of kidney failure, and/or heart failure, and/or pulmonary embolism, and/or deep venous thrombosis, and/or sepsis (Charlson Index $>3$ ). Presence of pregnancy or breast-feeding and/or history of other invasive malignancy in the previous 5 years were exclusion criteria.

Before random assignment, complete history and physical examination, routine hematology and biochemistry, electrocardiogram (ECG), chest x-ray or computed tomography (CT) scan, abdominal CT scan, and compilation of quality of life (QoL) questionnaires were required. Patients were considered eligible if disease at metastatic sites was supposed to be completely resectable based on imaging and clinical assessment. Eligible patients were triaged for staging laparoscopy to obtain histologic diagnosis and to provide the tumor load assessment through the PI score. Intra-operative inclusion criteria were: histologic diagnosis of epithelial ovarian cancer at frozen section, verified diffused intra-abdominal disease (PI score between 8 and 12 or high tumor load), and absence of mesenteric retraction ${ }^{5}$ (online supplemental figure S1). Patients were randomized at time of laparoscopy, when all inclusion criteria were met. If primary debulking surgery was chosen, open cytoreduction was performed at the same time.

\section{Study Treatments}

Selected patients were randomly assigned (1:1 ratio) to arm $A$ (standard treatment, primary debulking surgery) or arm B (experimental treatment, neoadjuvant chemotherapy).

In arm A, primary debulking surgery was performed with the aim of complete resection (R0). A secondary cytoreductive effort was not allowed in patients left with gross residual tumor (RT $>1 \mathrm{~cm})$.
Thereafter, six cycles of carboplatin 5 AUC and paclitaxel $175 \mathrm{mg} /$ $\mathrm{m}^{2}$ were planned (either every three weeks or weekly).

In arm B, neoadjuvant chemotherapy was administered as in arm A but only for three or four cycles. Patients with stable or responding disease underwent interval debulking surgery with the aim of complete debulking, after 3 or 4 weeks of rest. Chemotherapy was then continued to complete the planned six cycles, as in arm A. Interval debulking surgery was not allowed in patients having progressive disease under neoadjuvant chemotherapy, and chemotherapy was changed to a second line schedule. Progression of disease was assessed either by Response Evaluation Criteria In Solid Tumors (RECIST) or Gynecologic Cancer Intergroup (GCIG) criteria (either clinical or serological). ${ }^{78}$

Introduction of bevacizumab in combination with standard chemotherapy and as maintenance therapy was allowed in both arms following its approval in Italy (January 2014).

Patients with disease progression were considered not suitable for interval debulking surgery, and thus were not included in the post-operative complication analysis.

\section{Study Evaluations}

Tumor load before randomization was measured with the PI score named Fagotti's score. ${ }^{59}$ Response to chemotherapy and disease progression were defined according to RECIST criteria. ${ }^{710}$ CA125 serum levels were also registered as Gynecologic Cancer Intergroup (GCIG) criteria. ${ }^{811}$ Complexity of surgical procedures were graded according to the surgical complexity score. ${ }^{12}$ Severity of perioperative complications were graded on a 1 to 5 scale according to the Memorial Sloan Kettering Cancer Center Surgical Secondary Events Grading System. ${ }^{13}$ Patients were registered 30 days (early) and 6 months (late) after surgery. Post-operative complications were registered only if they did not exist before surgery. Sepsis was defined as the presence of bacteremia, and/or fever $\left(>38^{\circ} \mathrm{C}\right.$ for two consecutive times), and/or leucocytosis, all leading to poor organ function.

QoL was assessed using the EORTC QoL questionnaire (QLQC30 ) and the ovarian cancer-specific QoL questionnaire (QLQOv28). ${ }^{1415}$ Both questionnaires are designed to be compiled by the patient. They were completed at baseline, at the fourth cycle or before interval debulking surgery (in arm $A$ and $B$, respectively), at the sixth cycle, and 6 months after completion of chemotherapy. Patients were followed and monitored by clinical examination and CA125 measurements every 3 months for 2 years, every 6 months in the third year, then, in case of clinical disease absence, annually. Thorax and abdominal CT scans were performed every 3 months during treatment, and every 6 months during follow-up.

Progression-free survival was defined as the interval between date of randomization and date of progression (radiological or clinical assessed) or death, whichever occurred first, or date of last follow-up for patients alive and without progression. Overall survival was defined as the interval between date of randomization and date of death (or date of last follow-up for patients alive).

\section{Study Design}

SCORPION was an open-label, randomized phase III study. The primary endpoint was superiority of neoadjuvant chemotherapy followed by interval debulking surgery versus primary debulking surgery in terms of peri-operative morbidity. Subsequently, a 
second primary endpoint was added: superiority of neoadjuvant chemotherapy followed (or not) by interval debulking surgery versus primary debulking surgery in terms progression-free survival, and the sample size was enlarged accordingly. Reasons for superiority design lie in results of the EORTC 55971 trial. ${ }^{1}$ In a subgroup analysis of that study, neoadjuvant chemotherapy showed improved clinical outcome compared with primary debulking surgery in patients with stage IV disease versus stage IIIC. Conversely, longer survival was shown in primary debulking surgery versus neoadjuvant chemotherapy in patients with initial abdominal disease (largest size $<5 \mathrm{~cm}$ ). The rationale was that all cases with abdominal high tumor load have a similar prognosis independently from the presence of distant metastatic disease (both stage IIIC and IV).

Secondary endpoints included overall survival and QoL. Sample size calculation for the first co-primary endpoint (major perioperative morbidity) has been previously reported. ${ }^{4}$ Sample size calculation for the second co-primary endpoint (progression-free survival) was performed assuming a median progression-free survival of 12 months in the control arm (primary debulking surgery) as reported in the literature, ${ }^{12}$ and a 0.60 hazard ratio (HR) with neoadjuvant chemotherapy. Considering a type I error (two tailed) $\alpha=0.05$, a type II error $\beta=0.2$ (power $80 \%$ ), and a 10\% dropout rate, 166 patients were needed to detect 120 recurrences in the study population (EAST; Cytel Software, Cambridge, MA).

A computer-driven minimization procedure randomly assigned patients based on laparoscopic results to standard arm or experimental arm in a 1:1 ratio, at the Clinical Trial Unit of the Catholic University of the Sacred Heart, Fondazione Policlinico Universitario A. Gemelli IRCCS, Rome, Italy. Stratification factors were not anticipated. If available, the enrollment in other therapeutic trials was allowed in both arms since most had overall survival as the primary endpoint. After treatment completion, patients were followed for a minimum of 3 years.

\section{Data Analysis}

Baseline surgical and post-operative characteristics were presented as numbers (\%) for categorical variables and as mean (SD) for continuous variables. $\chi^{2}$ and Mann-Whitney $U$ tests were used in order to detect statistical differences according to the treatment arm, if appropriate. Median follow-up was calculated according to the inverted Kaplan-Meier technique. ${ }^{16}$ Overall and progressionfree survival curves were estimated by the Kaplan-Meier product limit method and compared by log-rank test. Cox proportional hazards models were used to assess the effect of clinically relevant factors such as treatment arms, age, ECOG performance status, stage, CA125 serum levels, and residual tumor at surgery on progression-free and overall survival, if applicable.

Compliance analyses were performed according to the perprotocol principle, including only patients receiving surgery. Efficacy analyses were performed according to the intention-to-treat principle: all randomly assigned patients were included regardless of whether they received all planned procedures or could be evaluated. No imputation was undertaken to deal with missing data and only complete cases were included in the analyses. All estimates were presented with two-sided $95 \% \mathrm{Cls}$, and differences were considered significant at a level of $p<0.05$. STATA statistical software program version 13.1 was used (Stata Corp, College Station, TX).

\section{RESULTS}

\section{Patient Characteristics}

The CONSORT (Consolidated Standards of Reporting Trials) diagram of the trial is reported in online supplemental figure S2. Between 0ctober 2011 and May 2016, 171 patients were randomly assigned. Eighty-four patients were assigned to primary debulking surgery (arm A), and 87 patients were assigned to neoadjuvant chemotherapy (arm B). Moreover, although the randomization was not stratified based on stage of disease, there is no statistical imbalance of stage IVB between the arms ( $8.3 \%$ vs $2.3 \%, p=0.20)$. Baseline characteristics are reported in Table 1.

\section{Treatment Compliance}

Information was not available for two patients who were lost during treatment, one for each arm. Of the remaining 169 patients, 26 patients did not complete treatment; reasons are shown in online supplemental figure S2. Finally, 143 of 171 (83.6\%) randomized patients received assigned treatment, 71 in arm $A$ and 72 in arm B. Overall, the median number of chemotherapy cycles was 6 (range 1-8) in arm $A$ and 6 (range 2-8) in arm $B(p=0.97)$. Ten patients received chemotherapy outside our hospital and some $(n=4)$ received eight cycles, adhering to their medical oncologists' decision. In arm B, the pre-operative median number of cycles was 4 (range 3-4). Carboplatin/paclitaxel, every 3 weeks, was the most common chemotherapy schedule employed (153 of 164 patients receiving chemotherapy, 93.3\%); bevacizumab was included in 31 $(39.7 \%)$ patients in arm $A$ and $42(48.8 \%)$ in arm $B(p=0.34)$.

Comparison of surgical data was performed according to perprotocol analysis, between 84 patients receiving primary debulking surgery and 74 patients who underwent interval debulking surgery, due to exclusion of eight progressions/deaths during neoadjuvant chemotherapy, three refusals, and one lost to follow-up. Rates of complete resection were statistically significantly different between the arms (R0 47.6\% in arm A vs $67.0 \%$ in arm B; $p=0.0001$ ). The main reasons precluding the achievement of complete cytoreduction (R0) are reported in Table 2. Among them, two of 171 patients $(1.1 \%)$ were described as unresectable due to retroperitoneal disease. In both cases (see Table 2) the disease wrapped the aorta and/or vena cava from the suprarenal to the common iliac vessels. Intra-operative consultation with the vascular surgeon, the anesthesiologist, and the gynecologic oncologic surgeon led to this decision.

The extent of surgery, as assessed by the surgical complexity score and upper abdominal procedures, was statistically significantly higher in arm A versus arm B. Primary debulking surgery was associated with significantly longer operative time and hospital stay compared with the interval debulking surgery group (Table 2). Operative and post-operative details for debulked patients are shown in online supplemental table S1. Two hundred and sixty-nine early post-operative complications were observed (Table 3). However, only $53(19.7 \%)$ were major complications, mainly in arm A than in arm $B(p<0.0001)$. Overall, the most frequent severe early postoperative complication was pleural effusion requiring drainage (27 of $53,51 \%$ ) (online supplemental table S2). Seven patients experienced more than one early serious (G3 to G5) adverse event after surgery. Finally, seven of $84(8.3 \%)$ patients in arm A died due to post-operative complications (three early and four late deaths, not related to progressive disease) compared with none in arm B 
Table 1 Baseline characteristics of randomly assigned patients

\begin{tabular}{|c|c|c|c|}
\hline & $\begin{array}{l}\text { Overall } \\
(n=171)\end{array}$ & $\begin{array}{l}\text { Arm A } \\
\text { PDS } \\
(n=84)\end{array}$ & $\begin{array}{l}\text { Arm B } \\
\text { NACT } \\
(n=87)\end{array}$ \\
\hline Characteristic & $\mathbf{N}(\%)$ & $\mathbf{N}(\%)$ & $\mathbf{N}(\%)$ \\
\hline Age (years), mean (SD) & $56.1(10.4)$ & $54.8(9.7)$ & $56.2(10.7)$ \\
\hline \multicolumn{4}{|l|}{ ECOG performance status } \\
\hline 0 & $79(46.2)$ & $40(47.6)$ & $39(44.8)$ \\
\hline 1 & $76(44.4)$ & $35(41.7)$ & $41(47.1)$ \\
\hline 2 & $16(9.4)$ & $9(10.7)$ & $7(8)$ \\
\hline CA125 serum level IU/ml, mean (SD) at diagnosis * & $2368.4(3199.3)$ & $2653.5(3944.2)$ & $2099.8(2278.6)$ \\
\hline \multicolumn{4}{|l|}{ Histotype } \\
\hline High grade serous carcinoma & $167(97.7)$ & $81(96.4)$ & $86(98.9)$ \\
\hline Low grade serous carcinoma & $2(1.2)$ & $1(1.2)$ & $1(1.1)$ \\
\hline Clear cell carcinoma & $1(0.6)$ & $1(1.2)$ & $0(0)$ \\
\hline Carcinosarcoma & $1(0.6)$ & $1(1.2)$ & $0(0)$ \\
\hline \multicolumn{4}{|l|}{ Grading } \\
\hline G1 & $2(1.2)$ & $1(1.2)$ & $1(1.1)$ \\
\hline G2 & $4(2.4)$ & $2(2.4)$ & $2(2.3)$ \\
\hline G3 & $164(96.5)$ & $80(96.4)$ & $84(96.6)$ \\
\hline na & 1 & 1 & 0 \\
\hline \multicolumn{4}{|l|}{ FIGO surgical stage (2014) } \\
\hline IIIC & $150(87.7)$ & $71(84.5)$ & $79(90.8)$ \\
\hline IV & $21(12.3)$ & $13(15.5)$ & $8(9.2)$ \\
\hline IVA & $12(7)$ & $6(7.1)$ & $6(6.9)$ \\
\hline IVB & $9(5.3)$ & $7(8.3)$ & $2(2.3)$ \\
\hline Mean PI score at diagnosis (SD) & $9.3(1.4)$ & $9.1(1.4)$ & $9.4(1.3)$ \\
\hline
\end{tabular}

*Information available for 169 patients: 82 in arm A, 87 in arm B.

ECOG, Eastern Cooperative Oncology Group; FIGO, International Federation of Gynecology and Obstetrics; NACT, neoadjuvant chemotherapy; PDS, primary debulking surgery; PI, predictive index.

$(p=0.006)$ (online supplemental table S3). Thirty-five late complications within 6 months after surgery were also observed in 11 patients: 10 in arm $A$ and one in arm $B(p=0.009)$. However, the difference of 13 major late post-operative complications in arm $A$ versus one in arm B was not statistically significant (Table 3).

\section{Efficacy}

All 171 patients were included in the intent to treat analyses. In March 2019, after a median follow-up of 59 months $(95 \% \mathrm{Cl} 53$ to 64$), 142(83 \%)$ disease progressions (69 in arm $A$ and 73 in arm B; $p=0.76)$ and $103(60.2 \%)$ deaths (47 in arm $A$ and 56 in arm $B ; p=0.26$ ) had occurred. Median progression-free survival was 15 months for patients assigned to primary debulking surgery compared with 14 months for patients assigned to neoadjuvant chemotherapy (HR 1.05, $95 \% \mathrm{Cl} 0.77$ to 1.44 ; log rank $\mathrm{p}=0.73$ ). Median overall survival was 41 months for patients assigned to primary debulking surgery and 43 months for patients assigned to neoadjuvant chemotherapy (HR 1.12, 95\% Cl 0.76 to 1.65; log rank $\mathrm{p}=0.56$ ). Progression-free and overall survival curves are shown in Figure 1. Five-year progression-free and overall survival were, respectively, $4.5 \%$ and $39.7 \%$ in arm $A$ and $7.5 \%$ and $31.8 \%$ in arm B.

Exploratory analysis of the study population, according to residual tumor, showed a significantly longer progression-free and overall survival in patients who underwent complete resection with respect to patients with residual tumor of $1-10 \mathrm{~mm}$ and any other residual or no cytoreduction (median progression-free survival: 16 months, 14 months, 10 months, 5 months, respectively, $p<0.0001$; median overall survival: 51 months, 40 months, 18 months, 10 months, respectively, $p<0.0001$ ) (online supplemental figure $\mathrm{S} 3$ ). However, in the subgroup analysis of patients with complete debulking, no statistically significant differences were found between primary debulking surgery versus interval debulking surgery treatment for both progression-free survival ( $\mathrm{HR} 1.01,95 \% \mathrm{Cl} 0.66$ to 1.55; $\mathrm{p}=0.96)$ and overall survival $(\mathrm{HR} 1.10,95 \% \mathrm{Cl} 0.61$ to $1.97 ; \mathrm{p}=0.74)$ (online supplemental figure S4).

At multivariable analysis for progression-free and overall survival, including treatment arm, age, ECOG performance status, stage, CA125 serum levels, and residual tumor at surgery, only 
Table 2 Operative details for debulked patients

\begin{tabular}{|c|c|c|c|c|}
\hline & $\begin{array}{l}\text { Overall } \\
(n=158)\end{array}$ & $\begin{array}{l}\text { Arm A } \\
\text { PDS } \\
(\mathrm{n}=84)\end{array}$ & $\begin{array}{l}\text { Arm B } \\
\text { NACT-IDS } \\
(n=74)\end{array}$ & \\
\hline Characteristic & $\mathbf{N}(\%)$ & $\mathbf{N}(\%)$ & $\mathbf{N}(\%)$ & $P$ value \\
\hline Size of residual disease & & & & 0.001 \\
\hline No gross & $97(61.4)$ & $40(47.6)$ & $57(77.0)$ & \\
\hline $1-10 \mathrm{~mm}$ & $54(34.2)$ & $38(45.2)$ & $16(21.6)$ & \\
\hline \multicolumn{5}{|l|}{ Reasons } \\
\hline Miliary carcinomatosis & $43(27.2)$ & $27(32.1)$ & $16(21.6)$ & \\
\hline Hepatic hilum & $6(3.8)$ & $6(7.1)$ & $0(0)$ & \\
\hline Retroperitoneal disease & $5(3.2)$ & $5(6.0)$ & $0(0)$ & \\
\hline$>10 \mathrm{~mm}$ & $7(4.4)$ & $6(7.1)$ & $1(1.4)$ & \\
\hline \multicolumn{5}{|l|}{ Reasons } \\
\hline Miliary carcinomatosis & $0(0)$ & $0(0)$ & $0(0)$ & \\
\hline Hepatic hilum & $4(2.5)$ & $4(4.8)$ & $0(0)$ & \\
\hline Retroperitoneal disease & $2(1.3)$ & $2(2.4)$ & $0(0)$ & \\
\hline Abdominal disease & $1(0.6)$ & $0(0)$ & $1(1.4)$ & \\
\hline No cytoreducion for progressive disease & $13 / 171(7.6)$ & $0 / 84(0)$ & $13 / 87(14.9)$ & \\
\hline Surgical complexity score ${ }^{9}$ & & & & $<0.0001$ \\
\hline 1 & $43(27.2)$ & $0(0)$ & $43(58.1)$ & \\
\hline 2 & $29(18.4)$ & $9(10.7)$ & $20(27.0)$ & \\
\hline 3 & $86(54.4)$ & 75 (89.3) & $11(14.9)$ & \\
\hline Upper abdominal procedures & & & & $<0.0001$ \\
\hline Yes & $111(70.3)$ & $83(98.8)$ & $28(37.8)$ & \\
\hline No & $47(29.7)$ & $1(1.2)^{\star}$ & $46(62.2)$ & \\
\hline Operative time (min), mean (SD) & 363.5 (145.3) & $460.6(102.6)$ & $253.2(101.4)$ & $<0.0001$ \\
\hline IO complications & $6(3.8)$ & $4(4.8)$ & $2(2.7)$ & 0.518 \\
\hline Vascular & $3(1.9)$ & $3(3.6)$ & $0(0)$ & \\
\hline Biliary duct & $1(0.6)$ & $1(1.2)$ & $0(0)$ & \\
\hline PTX after lung resection & $1(0.6)$ & $0(0)$ & $1(1.4)$ & \\
\hline Bladder injury & $1(0.6)$ & $0(0)$ & $1(1.4)$ & \\
\hline Mean hospital stay (days) (SD) & $11(9.5)$ & $14.8(11.3)$ & $6.7(3.9)$ & $<0.0001$ \\
\hline
\end{tabular}

Bold font highlights statistically significant differences.

*One patient did not undergo upper abdominal procedures because deemed unresectable at laparotomy.

IDS, interval debulking surgery; IO, intra-operative; NACT, neoadjuvant chemotherapy; PDS, primary debulking surgery; PTX, pneumothorax.

CA125 serum levels and residual tumor retained an independent prognostic value (Table 4).

\section{DISCUSSION}

In this phase III trial, interval debulking surgery was associated with significantly lower post-operative complication rates, including post-operative deaths, due to a less complex surgery. Moreover, neoadjuvant chemotherapy failed to show any progression-free survival advantage with respect to primary debulking surgery. Indeed, primary debulking surgery versus interval debulking surgery have similar efficacy (although the study was underpowered to detect a difference), and survival ranges were similar to other studies, with advanced surgical expertise, high patient tumor load, but exclusion of poly (ADP-ribose) polymerase (PARP) inhibitor maintenance treatment. ${ }^{17} 18$

Remarkably, lung complications, particular pleural effusion, were the more common complications compared with wound/abdominal infections or thromboembolic events in arm A; this outcome may be due to: (a) low median body mass index $\left(24.6 \mathrm{~kg} / \mathrm{m}^{2}\right.$; range 18.0 41.5); (b) very high incidence of diaphragmatic stripping/resection $(81 / 84=96.4 \%)$ performed in this population.

A possible explanation for the progression-free survival endpoint failure may be the increased use of upper abdominal procedures in our study population, allowing a complete/optimal cytoreduction in approximately $90 \%$ of cases, at primary debulking surgery. Based on the available data, it is conceivable that an increase in cytoreduction rates raises survival even in patients with high 
Table 3 Post-operative major complications

\begin{tabular}{|c|c|c|c|c|}
\hline \multirow[b]{2}{*}{ Characteristic } & $\begin{array}{l}\text { Overall } \\
(n=158)\end{array}$ & $\begin{array}{l}\text { Arm A } \\
\text { PDS } \\
(n=84) \\
\end{array}$ & $\begin{array}{l}\text { Arm B } \\
\text { NACT-IDS } \\
(n=74)\end{array}$ & \multirow[b]{2}{*}{$P$ value } \\
\hline & $\mathbf{N}(\%)$ & $\mathbf{N}(\%)$ & $\mathrm{N}(\%)$ & \\
\hline \multicolumn{5}{|l|}{ Early ( $\leq 30$ days) } \\
\hline Patients with major complications & $46(29.1)$ & $39(46.4)$ & $7(9.5)$ & $<0.0001$ \\
\hline All events & $269(100)$ & $177(65.8)$ & $92(34.2)$ & \\
\hline Major events & $53(19.7)$ & $46(25.9)$ & $7(7.6)$ & $<0.0001^{*}$ \\
\hline Grade III & $47(17.5)$ & $40(22.6)$ & $7(7.6)$ & 0.002 \\
\hline Grade IV & $3(1.1)$ & $3(1.7)$ & $0(0)$ & 0.209 \\
\hline Grade V & $3(1.1)$ & $3(1.7)$ & $0(0)$ & 0.2019 \\
\hline \multicolumn{5}{|l|}{ Late (1-6 months) } \\
\hline Patients with major complications & $11(7.0)$ & $10(11.9)$ & $1(1.4)$ & 0.009 \\
\hline All events & $35(100)$ & $31(88.6)$ & $4(11.4)$ & \\
\hline Major events & $14(40.0)$ & $13(41.9)$ & $1(25.0)$ & $0.515^{*}$ \\
\hline Grade III & $7(20.0)$ & $6(19.3)$ & $1(25.0)$ & 0.791 \\
\hline Grade IV & $3(8.6)$ & $3(9.7)$ & $0(0)$ & 0.588 \\
\hline Grade V & $4(11.4)$ & $4(12.9)$ & $0(0)$ & 0.455 \\
\hline
\end{tabular}

Bold font highlights statistically significant differences.

Rates are calculated on all events.

*Major (grades 3, 4, and 5) versus minor (grades 1 and 2) complications. ${ }^{10}$

IDS, interval debulking surgery; NACT, neoadjuvant chemotherapy; PDS, primary debulking surgery.

disease burden, thus resetting the difference in favor of neoadjuvant chemotherapy reported by the EORTC trial $^{1}$ and the individual patient meta-analysis of the EORTC and CHORUS trials ${ }^{3}$ in stage IV patients.

The prolonged median overall survival of up to 43 months reported in the SCORPION trial, in comparison with 27 months shown in the individual patient meta-analysis of the EORTC and CHORUS trials, ${ }^{3}$ seems to be the result of several trials with target-based drugs, performed during the study period on recurrent disease. ${ }^{19-23}$ It is also possible that the improved overall survival and progressionfree survival observed in the SCORPION trial reflects the selected study population that is significantly younger and has a better performance status compared with the EORTC/CHORUS trials.

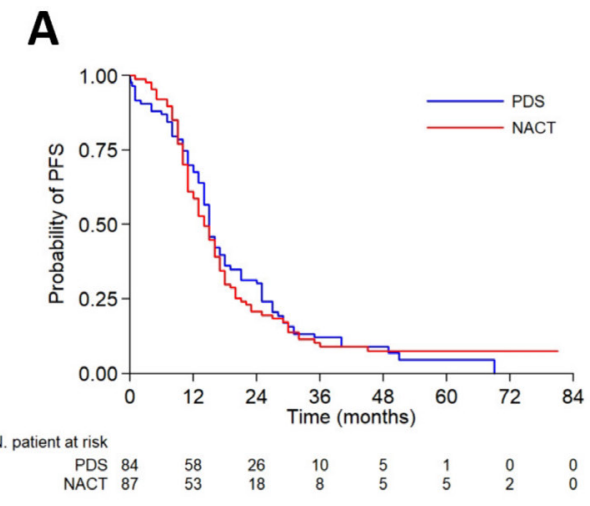

HR 1.05 ( $95 \% \mathrm{Cl}, 0.77$ to 1.44$)$; log rank $\mathrm{P}=.733$ )
Complete cytoreduction remains the most important prognostic factor in patients with advanced epithelial ovarian cancer encumbered with high tumor load. Interestingly, age, performance status, and stage are not associated with progression-free or overall survival (Table 4), which may be due to the very homogeneous series of cases. Moreover, our results confirm retrospective data suggesting that abdominal tumor dissemination and residual tumor are the only independent prognostic factors in these patients. ${ }^{9}$ Moreover, patients with R0 and R1-10 mm at primary debulking surgery have superimposable median progression-free survival (16 and 15 months, respectively) suggesting that patients with high tumor load, completely resected at the time of surgery, likely have micro/macroscopic unrecognized residual disease. Alternatively,

\section{B}

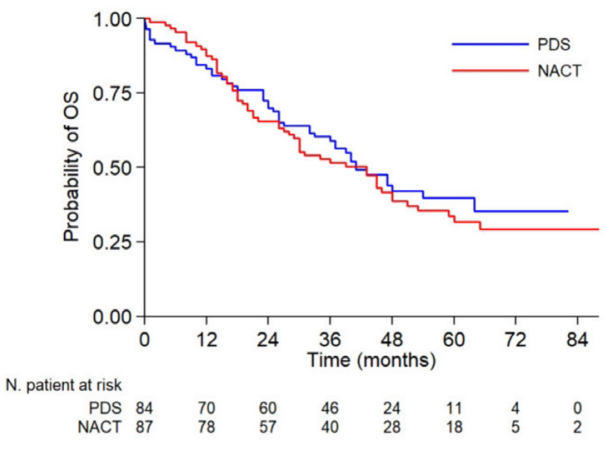

HR 1.12 (95\% Cl, 0.76 to 1.65 ); $\log$ rank $\mathrm{P}=.556$ )

Figure 1 Kaplan-Meyer plots for (A) progression-free survival (PFS) and (B) overall survival (OS) in the intention-to-treat population by study group. PDS, primary debulking surgery; NACT, neoadjuvant chemotherapy. 
Table 4 Cox proportional hazard model

\begin{tabular}{|c|c|c|c|c|c|c|c|c|c|c|c|c|}
\hline \multirow[b]{3}{*}{ Parameter } & \multicolumn{6}{|c|}{ Progession-free survival } & \multicolumn{6}{|c|}{ Overall survival } \\
\hline & \multicolumn{3}{|c|}{ Univariable analysis } & \multicolumn{3}{|c|}{ Multivariable analysis } & \multicolumn{3}{|c|}{ Univariable analysis } & \multicolumn{3}{|c|}{ Multivariable analysis } \\
\hline & HR & $95 \% \mathbf{C l}$ & $P$ value & HR & $95 \% \mathrm{Cl}$ & $P$ value & HR & $95 \% \mathrm{Cl}$ & $P$ value & HR & $95 \% \mathbf{C l}$ & $P$ value \\
\hline $\operatorname{Arm}(A$ vs $B)$ & 1.05 & 0.77 to 1.44 & 0.742 & 1.13 & 0.81 to 1.56 & 0.468 & 1.12 & 0.76 to 1.66 & 0.559 & 1.16 & 0.77 to 1.77 & 0.461 \\
\hline Age (continuous) & 1.00 & 0.99 to 1.02 & 0.590 & 1.00 & 0.98 to 1.01 & 0.841 & 1.01 & 0.99 to 1.03 & 0.427 & 1.00 & 0.98 to 1.02 & 0.841 \\
\hline $\begin{array}{l}\text { ECOG performance } \\
\text { status }\end{array}$ & 1.18 & 0.92 to 1.51 & 0.188 & 1.12 & 0.86 to 1.44 & 0.409 & 1.21 & 0.91 to 1.6 & 0.193 & 1.07 & 0.79 to 1.46 & 0.639 \\
\hline Stage (III vs IV) & 1.66 & 1.04 to 2.64 & 0.033 & 1.50 & 0.93 to 2.40 & 0.095 & 1.73 & 1.00 to 3.00 & 0.05 & 1.60 & 0.91 to 2.80 & 0.103 \\
\hline CA125 (continuous) & 1.00 & 1.00 to 1.00 & 0.003 & 1.00 & 1.00 to 1.00 & 0.025 & 1.00 & 1.00 to 1.00 & $<0.0001$ & 1.00 & 1.00 to 1.00 & 0.005 \\
\hline $\begin{array}{l}\text { Residual tumor at } \\
\text { surgery }\end{array}$ & 1.64 & 1.37 to 1.97 & $<0.0001$ & 1.56 & 1.29 to 1.88 & $<0.0001$ & 1.64 & 1.34 to 2.01 & $<0.0001$ & 1.51 & 1.22 to 1.86 & $<0.0001$ \\
\hline
\end{tabular}

$\mathrm{n}=169$ cases. Bold font indicates statistical significance.

$\chi^{2}$ of progression-free survival multivariable model 30.98. $\chi^{2}$ of overall survival multivariable model 28.62.

ECOG, Eastern Cooperative Oncology Group.

aggressive behavior of the disease and immune compromise induced by aggressive surgery may result in superimposable progression-free survival rates independently from residual tumor.

Regarding the role of staging laparoscopy and prediction of residual tumor, we previously published studies showing that a PI score $>8$ is associated with no chance of optimal resection (residual tumor $<1 \mathrm{~cm}$ ). ${ }^{9}$ Subsequently, we also demonstrated that the inclusion of upper abdominal procedures can reach the goal of complete resection in some patients with a PI score of $10{ }^{23}$ Therefore, the reported value of $47 \%$ as R0 is in line with these published data and, although the issue of correct selection of patients is still open and debated, ${ }^{24}$ we believe the SCORPION trial may add important information on this topic. Moreover, patients with RO at interval debulking surgery ( $67 \%$ of the neoadjuvant chemotherapy population) have a median progression-free survival of 16 months with much lower aggressive surgery (and related complications). Indeed, the choice of neoadjuvant chemotherapy also includes the possibility that primary chemoresistant patients may not benefit from potential effective surgery $(n=8,9.2 \%)$, highlighting the urgent need of molecular signatures for resistance to chemotherapy. On the other hand, this value is balanced with the high number of deaths/ progressions observed early after primary debulking surgery and during adjuvant treatment (within 3 months) ( $n=8,9.5 \%)$.

The unique design of this study is not comparable with the ongoing TRUST trial (NCT02828618). Indeed, although the latter involves centers with high surgical skills, all patients with presumed advanced ovarian cancer are randomized irrespective of their tumor load. Similarly, several differences can be highlighted with respect to the Japan Clinical Oncology Group (JCOG) 0602 trial, ${ }^{2526}$ such as the fact that only $12 \%$ of patients in the primary debulking surgery arm had primary complete cytoreduction, and also that one third of the remaining patients had an additional debulking surgery.

Our study has a number of limitations, mainly related to lack of statistical power to detect a minimal difference in term of months of survival. Although our study population was calculated with an HR of 0.60 , corresponding to an 8 month increase in median progressionfree survival from 12 to 20 months, with the 142 events collected for the analysis, the study had $66 \%$ power to detect a $0.67 \mathrm{HR}$, corresponding to a 6 month increase in median progression-free survival from 12 to 18 months. Power was significantly lower
$(40 \%)$ to detect a 4 month difference in median progression-free survival. We may argue that the aim of this study was to assess a "real" clinical benefit in terms of survival to be balanced with the perioperative complication rate after a large aggressive surgery. It might be useful adding data from the present study together with those already collected in the individual patient data meta-analysis by Vergote et al. ${ }^{3}$ This would allow increasing statistical power to detect a smaller but still clinically significant benefit, and to test interaction between treatment efficacy and patient characteristics. ${ }^{27}$ The imbalance in use of bevacizumab did not reach any statistically significant difference and thus should not be considered as a limitation. Finally, the single center design can be considered a strength on one side, thanks to the homogeneity of treatment and completeness of data, but it is difficult to generalize.

We consider that our study may be hypothesis generating, regarding the potential role of primary debulking surgery in a selected group of patients with high tumor load. Indeed, results suggest that the two approaches, neoadjuvant chemotherapy and primary debulking surgery, have the same efficacy but are associated with different toxicities.

\section{Author affiliations}

${ }^{1}$ Woman, Child and Public Health Department, Fondazione Policlinico Universitario A. Gemelli, IRCCS, Roma, Italy

${ }^{2}$ Università Cattolica del Sacro Cuore Sede di Roma, Roma, Italy

${ }^{3}$ Statistics Technology Archiving Research (STAR) Center, Fondazione Policlinico Universitario A. Gemelli IRCCS, Roma, Italy

${ }^{4}$ Department of Gynecologic Oncology, ARNAS Civico Di Cristina Benfratelli,

Palermo, Italy

${ }^{5}$ Gynecologic Oncology, University of Palermo, Palermo, Italy

${ }^{6}$ Gynecologic Oncology, Gemelli Molise spa, Università Cattolica del Sacro Cuore, Campobasso, Italy

\section{Twitter Anna Fagotti @annafagottimd}

Contributors The first author wrote the initial draft of the manuscript. All the authors contributed to subsequent revisions of the draft, agreed to submit the manuscript for publication, and vouch for the accuracy and completeness of the data and analyses, and for the fidelity of the trial to the protocol.

Funding This work was supported by Fondazione Policlinico Universitario A. Gemelli, IRCCS, Rome, Italy (nr 5750827).

Competing interests None declared.

Patient consent for publication Obtained. 


\section{Original research}

Provenance and peer review Not commissioned; externally peer reviewed.

Data availability statement Data have been collected prospectively on RedCapbased CRF. Data are available at the URL https://redcap-irccs.policlinicogemelli.it/. Only people officially authorized from the principal investigator can access data. The request should be addressed by email at anna.fagotti@policlinicogemelli.it or giovanni.scambia@policlinicogemelli.it.

\section{ORCID iD}

Anna Fagotti http://orcid.org/0000-0001-5579-335X

\section{REFERENCES}

1 Vergote I, Tropé CG, Amant F, et al. Neoadjuvant chemotherapy or primary surgery in stage IIIC or IV ovarian cancer. N Engl J Med 2010;363:943-53.

2 Kehoe S, Hook J, Nankivell M, et al. Primary chemotherapy versus primary surgery for newly diagnosed advanced ovarian cancer (CHORUS): an open-label, randomised, controlled, non-inferiority trial. Lancet 2015;386:249-57.

3 Vergote I, Coens C, Nankivell M, et al. Neoadjuvant chemotherapy versus debulking surgery in advanced tubo-ovarian cancers: pooled analysis of individual patient data from the EORTC 55971 and CHORUS trials. Lancet Oncol 2018;19:1680-7.

4 Fagotti A, Ferrandina G, Vizzielli G, et al. Phase III randomised clinical trial comparing primary surgery versus neoadjuvant chemotherapy in advanced epithelial ovarian cancer with high tumour load (SCORPION trial): final analysis of peri-operative outcome. Eur J Cancer 2016;59:22-33.

5 Fagotti A, Vizzielli G, De laco P, et al. A multicentric trial (OlympiaMITO 13) on the accuracy of laparoscopy to assess peritoneal spread in ovarian cancer. Am J Obstet Gynecol 2013;209:462. e1-462.e11.

6 Drinka PJ, Langer E. The Cockroft-Gault formula. J Am Geriatr Soc 1989;37:820.

7 Therasse P, Arbuck SG, Eisenhauer EA, et al. New guidelines to evaluate the response to treatment in solid tumors. European Organization for Research and Treatment of Cancer, National Cancer Institute of the United States, National Cancer Institute of Canada. J Natl Cancer Inst 2000;92:205-16.

8 Rustin GJ, Nelstrop AE, McClean P, et al. Defining response of ovarian carcinoma to initial chemotherapy according to serum CA 125. J Clin Oncol 1996;14:1545-51.

9 Vizzielli G, Costantini B, Tortorella L, et al. Influence of intraperitoneal dissemination assessed by laparoscopy on prognosis of advanced ovarian cancer: an exploratory analysis of a single-institution experience. Ann Surg Oncol 2014;21:3970-7.

10 Eisenhauer EA, Therasse P, Bogaerts J, et al. New response evaluation criteria in solid tumours: revised RECIST guideline (version 1.1). Eur J Cancer 2009;45:228-47.

11 Rustin GJ, Marples M, Nelstrop AE, et al. Use of CA-125 to define progression of ovarian cancer in patients with persistently elevated levels. J Clin Oncol 2001;19:4054-7.

12 Aletti GD, Dowdy SC, Podratz KC, et al. Relationship among surgical complexity, short-term morbidity, and overall survival in primary surgery for advanced ovarian cancer. Am J Obstet Gynecol 2007;197:676.e1-676.e7.
13 Strong VE, Selby LV, Sovel M, et al. Development and assessment of Memorial Sloan Kettering Cancer Center's surgical secondary events grading system. Ann Surg Oncol 2015;22:1061-7.

14 Aaronson NK, Ahmedzai S, Bergman B, et al. The European Organization for Research and Treatment of Cancer QLQ-C30: a quality-of-life instrument for use in international clinical trials in oncology. J Natl Cancer Inst 1993;85:365-76.

15 Greimel E, Bottomley A, Cull A, et al. An international field study of the reliability and validity of a disease-specific questionnaire module (the QLQ-OV28) in assessing the quality of life of patients with ovarian cancer. Eur J Cancer 2003;39.

16 Kaplan EL, Meier P. Nonparametric estimation from incomplete observations. J Am Stat Assoc 1958;53:457-81.

17 Horowitz NS, Miller A, Rungruang B, et al. Does aggressive surgery improve outcomes? Interaction between preoperative disease burden and complex surgery in patients with advancedstage ovarian cancer: an analysis of GOG 182. J Clin Oncol 2015;33:937-43.

18 Gasimli K, Braicu El, Richter R, et al. Prognostic and predictive value of the peritoneal cancer index in primary advanced epithelial ovarian cancer patients after complete cytoreductive surgery: study of tumor bank ovarian cancer. Ann Surg Oncol 2015;22:2729-37.

19 Pujade-Lauraine E, Ledermann JA, Selle F, et al. Olaparib tablets as maintenance therapy in patients with platinum-sensitive, relapsed ovarian cancer and a BRCA1/2 mutation (SOLO2/ENGOT-Ov21): a double-blind, randomised, placebo-controlled, phase 3 trial. Lancet Oncol 2017;18:1274-84.

20 Mirza MR, Monk BJ, Herrstedt J, et al. Niraparib maintenance therapy in platinum-sensitive, recurrent ovarian cancer. N Engl J Med 2016;375:2154-64.

21 Coleman RL, Oza AM, Lorusso D, et al. Rucaparib maintenance treatment for recurrent ovarian carcinoma after response to platinum therapy (ARIEL3): a randomised, double-blind, placebo-controlled, phase 3 trial. Lancet 2017;390:1949-61.

22 Ledermann JA, Harter P, Gourley C, et al. Overall survival in patients with platinum-sensitive recurrent serous ovarian cancer receiving olaparib maintenance monotherapy: an updated analysis from a randomised, placebo-controlled, double-blind, phase 2 trial. Lancet Oncol 2016;17:1579-89.

23 Petrillo M, Vizzielli G, Fanfani F, et al. Definition of a dynamic laparoscopic model for the prediction of incomplete cytoreduction in advanced epithelial ovarian cancer: proof of a concept. Gynecol Oncol 2015;139:5-9.

24 Vizzielli G, Costantini B, Tortorella L, et al. A laparoscopic riskadjusted model to predict major complications after primary debulking surgery in ovarian cancer: a single-institution assessment. Gynecol Oncol 2016;142:19-24.

25 Onda T, Satoh T, Saito T, et al. Comparison of survival between upfront primary debulking surgery versus neoadjuvant chemotherapy for stage III/IV ovarian, tubal and peritoneal cancers in phase III randomized trial: JCOG0602. J Clin Oncol 2018;36:5500-1.

26 Onda T, Satoh T, Ogawa G, et al. Comparison of survival between primary debulking surgery and neoadjuvant chemotherapy for stage III/IV ovarian, tubal and peritoneal cancers in phase III randomised trial. Eur J Cancer 2020;130:114-25.

27 Piedbois P, Buyse M. Meta-analyses based on abstracted data: a step in the right direction, but only a first step. J Clin Oncol 2004;22:3839-41. 